\title{
Microinjection of Cocaine into the Nucleus Accumbens Elicits Locomotor Activation in the Rat
}

\author{
J. M. Delfs, L. Schreiber, and A. E. Kelley \\ Department of Psychology, Harvard University, Cambridge, Massachusetts 02138
}

\begin{abstract}
Cocaine is believed to exert its psychostimulant effects through activation of the mesocorticolimbic system. Although the nucleus accumbens, in particular, has been hypothesized as the site of action of cocaine's stimulating effects, there is no direct evidence that microinjection of cocaine into this region produces behavioral activation. The present experiments investigated the locomotor response to microinjection of cocaine $(0,10,30,100 \mu \mathrm{g} / 0.5 \mu \mathrm{l})$ into the nucleus accumbens in rats. Cocaine elicited a pronounced, dose-dependent motor activation of approximately 60 min duration. This stimulant effect was blocked by prior administration of a dopamine (DA) receptor antagonist, cisflupenthixol. The response to cocaine was differentiated from nucleus accumbens microinjections of procaine and lidocaine, compounds that have potent local anesthetic effects but little affinity for the dopamine-uptake site. Neither procaine nor lidocaine $(0,10,30,100 \mu \mathrm{g} / 0.5 \mu \mathrm{l})$ had any overall effect, although activity was somewhat decreased in the initial part of the test session and increased at the end, relative to control activity. Cocaine injected into the anterior dorsal or ventrolateral striatum $(100 \mu \mathrm{g})$ also increased motor activity; procaine and lidocaine had no effect. Cocaine injected into the ventrolateral striatum significantly increased stereotypy. The amplitude of motor activation following cocaine injection into nucleus accumbens was much greater than that elicited at the other striatal sites. Further, observation of the time course of motor activation following cocaine injection into the anterior dorsal and ventrolateral striatum suggested that the motor effect was due to diffusion, most likely to the nucleus accumbens. The results show that cocaine can be microinjected with a considerable degree of specificity, and they support the hypothesis that the nucleus accumbens is the major site of cocaine's stimulant action.
\end{abstract}

The psychostimulant effects of cocaine have been extensively studied and appear to be mediated, at least in part, by the mesocorticolimbic dopamine (DA) system (Roberts et al., 1977, 1980; Roberts and Koob, 1982; Goeders and Smith, 1983, 1986; Post et al., 1987). A number of studies have shown that cocaine is a potent blocker of the presynaptic DA uptake site (Reith et al., 1980; Ritz et al., 1987) and therefore increases synaptic

\footnotetext{
Received May 12, 1989; revised July 10, 1989; accepted July 11, 1989.

This work was supported by grant 5 R29 DA04788 from the National Institute on Drug Abuse, and by Harvard University, Faculty of Arts and Sciences.

Correspondence should be addressed to J. M. Delfs, Department of Psychology, Harvard University, 33 Kirkland St., Cambridge, MA 02138.

Copyright (C) 1990 Society for Neuroscience $0270-6474 / 90 / 010303-08 \$ 02.00 / 0$
}

levels of DA. Although cocaine also binds to other uptake sites, it has recently been shown that a close correlation exists between the ability of cocaine-like compounds to bind to the DA uptake site and their potency in self-administration paradigms (Ritz et al., 1987). DA is known to be critical for an animal's motivational arousal and behavioral activation, and the mesolimbic system has been directly implicated in the control of spontaneous locomotor behavior (Kelly et al., 1975; Pijnenburg et al., 1976). Pcripheral injection of cocaine increases locomotor activity and this effect is mediated by DA (review, Post et al., 1987). This hypothesis was originated by Kelly and Iversen (1976), who reported that selective lesioning of DA fibers in the nucleus accumbens substantially reduced cocaine-induced locomotor behavior.

Intravenous self-administration of cocaine, as well as many other rewarding substances, has been shown to be readily acquired and maintained in rats (Pickens and Thompson, 1968; Schuster and Thompson, 1969; Collins et al., 1984). Selective destruction of DA nerve terminals in the nucleus accumbens with 6-hydroxydopamine (6-OHDA) or kainic acid lesions reduces or extinguishes intravenous self-administration of cocaine in rats (Roberts et al., 1980; Pettit et al., 1984; Zito et al., 1985). This finding demonstrates that the removal of DA, specifically in the mesocorticolimbic pathway, prevents cocaine from producing its rewarding effects.

The use of intracerebral microinjections has contributed significantly to our understanding of the relative roles of specific anatomical sites of neurotransmitters in behavior. However, despite widespead use of localized injections of many drugs, there have been few attempts to investigate the behavioral effects of localized cocaine injections. The general reluctance of researchers to inject cocaine directly in the brain is due to the inherent properties of the drug itself. Cocaine, in addition to being a psychomotor stimulant, is also a powerful local anesthetic. For this reason, it would be difficult to interpret behavioral changes as due to the stimulant or anesthetic nature of the drug. Further, cocaine is extremely lipophilic, diffusing rapidly through body tissues. Researchers may therefore believe that restricted injections into discrete regions of the brain are difficult to achieve.

In the present study an attempt was made to overcome the difficulties inherent in cocaine microinjections by utilizing several strategies to address the above criticisms. First, in order to clarify which of cocaine's properties is responsible for the behavioral effects, comparisons were made with 2 other drugs, procaine and lidocaine. Both are local anesthetics, similar to cocaine in chemical structure, but are not abused substances. Second, in order to ascertain the site specificity of cocaine's effects and the degree to which it diffuses, 3 separate sites within 
the forebrain DA system were studied. Previous work has found that amphetamine, which is also lipophilic, produced differential behavioral effects when infused into different areas of the striatum (Carr and White, 1987; Kelley and Gauthier, 1987; Kelley et al., 1989). Additionally, in order to minimize diffusion, a small injection volume was utilized $(0.5 \mu \mathrm{l})$.

The nucleus accumbens was chosen as the primary test site because of the large body of research implicating it in the initiation of locomotor activity. The ventrolatcral striatum and the anterior dorsal striatum were chosen because they are high in DA content, are relatively distant from the nucleus accumbens, and are generally not associated with locomotor activity. The purpose of the experiment was to observe general motor activity following microinjection of cocaine, procaine, and lidocaine and to observe any dissociable differences between the drugs or sites used.

\section{Materials and Methods}

Animals and surgery. Male Sprague-Dawley rats (total $n=53$, Charles River, Wilmington, MA) weighing 300-370 gm were used throughout the experiments. Upon arrival, animals were housed individually in clear plastic cages with wire grid floors. Subjects were maintained on a 12:12-hr light/dark cycle (lights on from 08:00 to 20:00) with food and water available ad libitum. Behavioral testing was performed between 10:00 A.M. and 4:00 P.M., during the light cycle. For implantation of cannulae, animals were anesthetized with Nembutal $(1 \mathrm{ml} / \mathrm{kg}$, i.p.) and placed in a Kopf stereotaxic frame with the incisor bar set $5.0 \mathrm{~mm}$ above the interaural line. Bilateral stainless steel cannulae guides (23 gauge) were implanted using standard stereotaxic procedures. Guides were aimed at 3 brain sites: the nucleus accumbens (N. Acc.), the ventrolateral striatum (VLS), and the anterior dorsal striatum (ADS). For implantation in the nucleus accumbens $(n=39)$, the coordinates were $3.5 \mathrm{~mm}$ anterior to bregma, $1.7 \mathrm{~mm}$ lateral to the midline, and $5.7 \mathrm{~mm}$ ventral from the skull. For implantation in the ventrolateral striatum $(n=7)$, the coordinates were $2.0 \mathrm{~mm}$ anterior to bregma, $4.0 \mathrm{~mm}$ lateral to the midline, and $4.7 \mathrm{~mm}$ ventral from the skull. For implantation in the anterior dorsal striatum $(n=7)$, the coordinates were $3.1 \mathrm{~mm}$ anterior to bregma, $3.0 \mathrm{~mm}$ lateral to the midline, and $3.5 \mathrm{~mm}$ ventral from the skull. Cannula guides were affixed to the skull with dental acrylic cement and stainless steel screws. Wire stylets were placed in the guides to prevent occlusion. Animals were allowed $3 \mathrm{~d}$ for recovery prior to behavioral testing.

Drugs and microinfusion. The drugs used in the following experiments were cocaine hydrochloride (Sigma, St. Louis, MO), procaine hydrochloride (Sigma), and lidocaine hydrochloride (Astra Pharmaceuticals, Westboro, MA). All drugs were dissolved in 0.9\% sterile saline. For within-subjects experiments, all doses or treatments and a vehicle (saline) were administered in a counterbalanced order, depending on experimental protocol, with a minimum of $48 \mathrm{hr}$ between each injection. The infusions were bilateral in a volume of $0.5 \mu \mathrm{l} / \mathrm{side}$. In one experiment, pretreatment with cis-flupenthixol (Lundbeck, Copenhagen, Denmark) or trans-flupenthixol $(0.3 \mathrm{mg} / \mathrm{kg})$ was administered intraperitoneally $30 \mathrm{~min}$ prior to intracerebral microinjection.

Prior to injection, the stylets were removed and the cannulae guides were cleared with a square dental broach. Bilateral injector cannulae (30 gauge) were lowered through the guides to the site of stimulation. The final coordinates were $8.2 \mathrm{~mm}, 7.2 \mathrm{~mm}$, and $5.5 \mathrm{~mm}$ ventral from the skull for the N. Acc., the VLS, and the ADS, respectively. The injector cannulae were connected via polyethylene tubing (PE-10, Clay Adams) to a microdrive pump (Harvard Apparatus) which controlled the infusion. The animals were held loosely by the experimenter while the infusion was delivered over $93 \mathrm{sec}$. A 1 -min period was allowed for diffusion before removal of the injection cannulae and replacement of the stylets.

Behavioral design. This study consisted of 2 experiments. In Experiment 1,39 rats had cannulae guides implanted in the nucleus accumbens. Twenty-four of these rats were randomly divided into 4 groups of 6 rats (between-subjects design). Each group was infused with 10, 30, or $100 \mu \mathrm{g}$ cocaine or saline vehicle. Following this testing, 12 of the 24 rats were selected randomly and assigned to 2 new groups. Six rats were given $0.3 \mathrm{mg} / \mathrm{kg}$ of the DA antagonist cis-flupenthixol, intraperitoneally (i.p.), $30 \mathrm{~min}$ prior to a nucleus accumbens microinjection of $100 \mu \mathrm{g}$ of cocaine. The other 6 animals were treated with $0.3 \mathrm{mg} / \mathrm{kg}$ i.p. of transflupenthixol, the inactive isomer, $30 \mathrm{~min}$ prior to an identical cocaine injection. For the experiment with procaine, rats $(n=7)$ were infused with $10,30,100 \mu \mathrm{g}$ of procaine or saline vehicle counterbalanced for order in a within-subjects design. For the experiment with lidocaine, rats $(n=8)$ were administered lidocaine $(10,30,100 \mu \mathrm{g})$ or saline vehicle in a manner similar to that of the procaine expcriment.

In Experiment 2, rats with cannulae guides implanted in the ADS ( $n$ $=7$ ) were administered 4 counterbalanced injections of saline, $100 \mu \mathrm{g}$ of cocaine, $100 \mu \mathrm{g}$ of procaine, or $100 \mu \mathrm{g}$ of lidocaine. A separate group of rats $(n=7)$ received the same infusions directly into the VLS.

Apparatus and testing procedure. Animals were tested immediately following microinjection in an automated activity testing apparatus. The apparatus consisted of transparent plastic cages similar to the animals' home cages, each of which was equipped with 2 photoelectric cells along the long axis of the cage. The cells were interfaced with a computer that automatically recorded the number of times the photoelectric beam was interrupted. Total activity counts (total beam breaks) were printed out every $5 \mathrm{~min}$ for $60 \mathrm{~min}$. The computer also provided a measure of locomotion throughout the cage. In this measure, a beam interruption was counted only if it was preceded by a beam interruption on the other photocell. A measure of stereotypy was determined for the $100-\mu \mathrm{g}$ dose of cocaine in each of the 3 sites by dividing the total activity scores by the locomotion scores. The resulting ratio established an indicator of perseverative movement (stereotypy). Animals were habituated to the activity cages for $3 \mathrm{~d}$ prior to testing $(2 \mathrm{hr} / \mathrm{d})$ and for $1 \mathrm{hr}$ on each test day, prior to microinfusion.

Histology. At the end of the experiments, rats were deeply anesthetized with Nembutal ( $2 \mathrm{ml} / \mathrm{kg}, \mathrm{i} . \mathrm{p}$.) and perfused transcardially with isotonic saline followed by $10 \%$ formalin. Brains were removed and stored in formalin for subsequent cutting. Brain sections ( $60 \mu \mathrm{m}$ thickness) were mounted on slides, defatted, and stained with cresyl violet. Placement of cannulae tracks was verified by examination under a tissue projector, and selected sections were photographed.

Statistics. All data were analyzed using an IBM-compatible statistical software program (CRISP Interactive Statistical Package). The data were subjected to analysis of variance, followed by Newman-Keuls comparisons between means for dose or treatment effects, or by analysis of simple main effects for interactions (Bruning and Kintz, 1977).

\section{Results}

\section{Experiment $1-$ nucleus accumbens}

Cocaine administration into the nucleus accumbens induced marked enhancement of motor activity at all 3 doses. This dosedependent increase in activity is shown in Figure $1 \mathrm{~A}$. An overall significant effect of dose was obtained $(F(3,20)=5.3, p<0.01)$, as well as a dose $\times$ time interaction $(F(33,220)=2.64, p<$ $0.001)$. Total activity following the $100-\mu \mathrm{g}$ dose was significantly higher than that following saline (Newman-Keuls, $p<0.01$ ), as it was for the $30-\mu \mathrm{g}$ and $10-\mu \mathrm{g}$ doses $(p<0.05)$. All 3 doses produced significant dose $\times$ time interactions with respect to saline $(100-\mu \mathrm{g}$ dose, $F(11,220)=4.6, p<0.001 ; 30-\mu \mathrm{g}$ dose, $F(11,220)=1.76, p<0.05 ; 10-\mu \mathrm{g}$ dose, $F(11,220)=1.75, p<$ 0.05 ). The time course (Fig. $1 B$ ) shows that all 3 doses of cocaine elicited an increase in activity during the early part of the test session, which was sustained for $35 \mathrm{~min}$ after the $10-\mu \mathrm{g}$ dose, 45-50 min for the 30- $\mu \mathrm{g}$ dose, and $55 \mathrm{~min}$ for the $100-\mu \mathrm{g}$ dose. In contrast, activity levels following saline treatment declined quite rapidly after infusion.

The effect of pretreatment with cis-flupenthixol on activity induced by cocaine injection into the nucleus accumbens is shown in Figure 2. Cis-flupenthixol significantly reduced the level of activation induced by the $100-\mu \mathrm{g}$ injection of cocaine $(F(1,10)=5.26, p<0.044)$. This can be contrasted with the activity levels observed in the group treated with trans-flupen- 
A. Total actlvity - N.Acc.

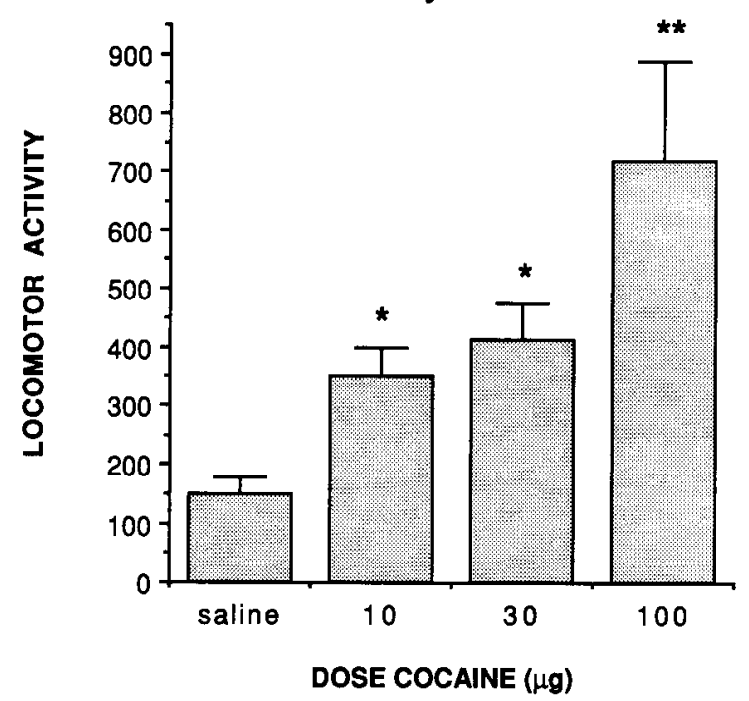

B. Time course - N.Acc.

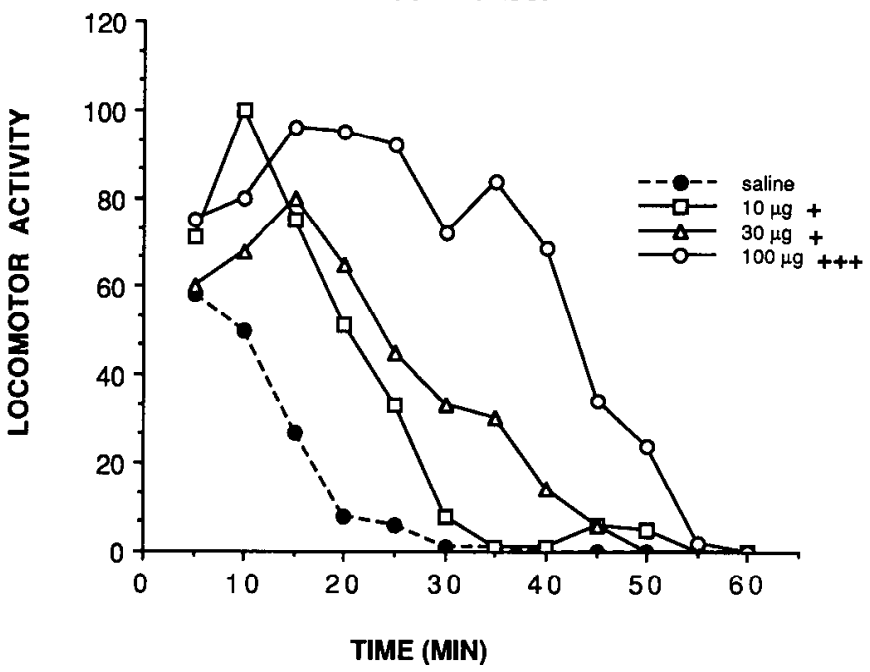

Figure 1. A, Total locomotor activity following microinjection of cocaine into the nucleus accumbens. ${ }^{*} p<0.05,{ }^{* *} p<0.01$. B. Pattern of locomotor activity over time, following nucleus accumbens injection of cocaine.,$+ p<0.05$, dose $\times$ time interaction with respect to saline.

thixol, which were similar to levels following the $100-\mu \mathrm{g}$ cocaine infusion in the first experiment.

There was no overall effect on activity following administration of procaine into the nucleus accumbens (Fig. $3 A$ ). There was, however, a significant overall dose $\times$ time interaction $(F(33,198)=1.58, p<0.05)$. Further analysis revealed that the time course of activity was significantly different from saline following both the $30-\mu \mathrm{g}$ dose $(F(11,198)=2.25, p<0.025)$ and the $100-\mu \mathrm{g}$ dose $(F(11,198)=2.36, p<0.01)$. The time course following procaine administration into the nucleus accumbens is shown in Figure $3 B$. It can be observed that rats treated with the highest doses of procaine are less active in the initial part of the session, but become somewhat more active than control toward the middle of the session.

Lidocaine administration produced no overall dose effect, but did elicit a significant dose $\times$ time interaction $(F(33,231)=$ $1.74, p<0.01)$. Further analysis revealed that this effect was
Total activity - N.Acc.

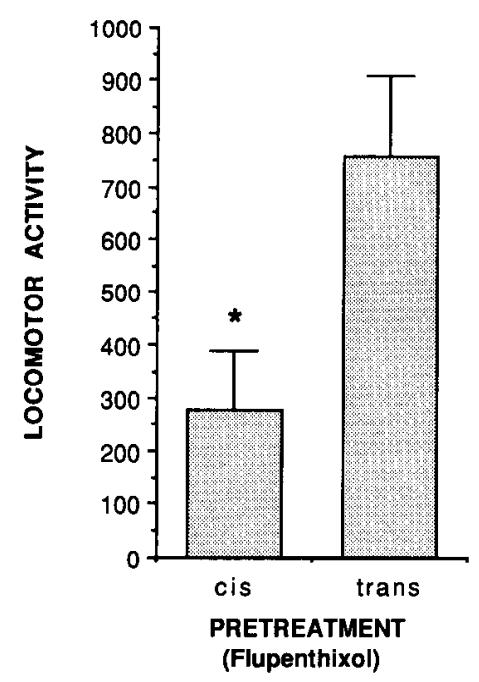

Figure 2. Total locomotor activity following microinjection of cocaine $(100 \mu \mathrm{g})$ into nucleus accumbens, in rats pretreated with cis- or transflupenthixol $(0.3 \mathrm{mg} / \mathrm{kg}$ i.p. $)$

due to an interaction between saline and the highest dose of lidocaine $(F(11,231)=3.79, p<0.001)$. Total activity and the time course for lidocaine are present in Figure $4 A$ and $B$. It can be observed from the time course that activity following the highest dose of lidocaine was very low initially and higher later in the session, compared with saline. Comparison of thesc 2 groups over individual time points indicated that at $15 \mathrm{~min}$ the lidocaine-treated rats were significantly less active than control $(p<0.01)$, and that at 35 and $40 \mathrm{~min}$ they were somewhat more active, although not statistically significantly $(p<0.06)$.

\section{Experiment 2-Anterior dorsal and ventrolateral striatum}

In the first part of this experiment, the effects of $100 \mu \mathrm{g}$ cocaine, procaine, and lidocaine injected into the anterior dorsal striatum were compared. Analysis of total activity scores indicated a significant overall treatment effect $(F(3,18)=9.85, p<0.001)$, due to the cocaine treatment $(p<0.01)$. Figure $5 \mathrm{~A}$ shows that cocaine injected into the ADS elicited an increase in activity. There was also a significant overall treatment $\times$ time interaction $(F(33,198)=1.82, p<0.01)$, due to an effect of cocaine on time course $(F(11,198)=4.3, p<0.001)$. It can be observed from Figure $5 B$ that animals treated with cocaine in the ADS are less active initially, and become more active later in the session. In contrast to cocaine, neither lidocaine nor procaine significantly affected activity.

Analysis of activity scores following infusion of cocaine, lidocaine, or procaine into the ventrolateral striatum indicated a significant overall treatment effect $(F(3,18)=8.46, p<0.001)$. Post hoc comparisons of means revealed that cocaine-induced activity was significantly greater than that following saline infusion $(p<0.01)$, as shown in Figure $5 C$. There was also a significant treatment $\times$ time interaction $(F(33,198)=2.98, p$ $<0.001)$, due to an interaction between saline scores and cocaine scores $(F(11,198)=4.92, p<0.001)$. The time course of activity is shown in Figure $5 D$. It is clear from this figure that the motor activity of cocaine-treated rats was highest toward the middle of the session. Informal observation of the rats following cocaine administration revealed high levels of stereo- 


\section{A. Total activity - N.Acc.}

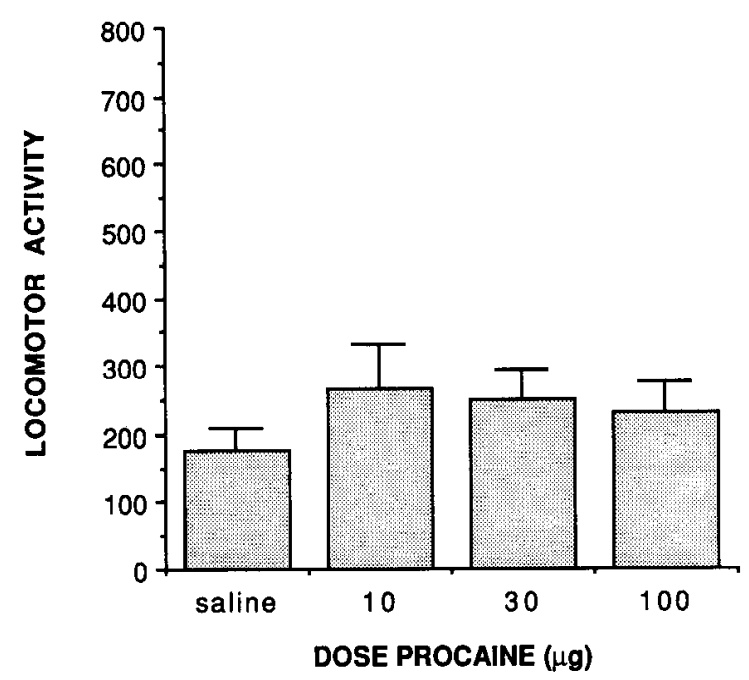

B. Time course - N.Acc.

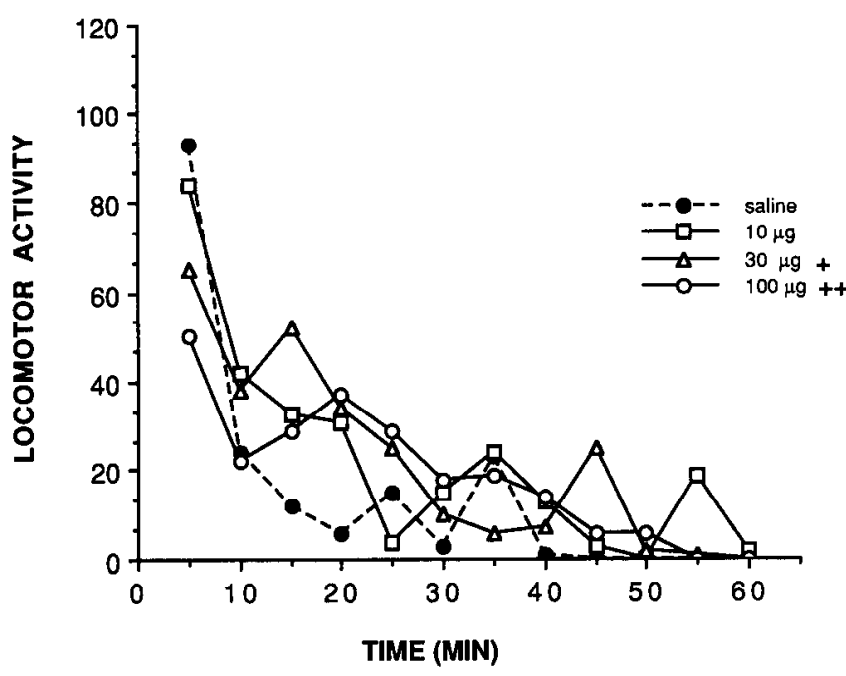

Figure 3. A, Total locomotor activity following microinjection of procaine into nucleus accumbens. $B$, Pattern of locomotor activity over time, following nucleus accumbens injection of procaine.,$+ p<0.05$, ,$++ p<0.01$, dose $\times$ time interaction with respect to saline.

typed, head-down sniffing rather than locomotion throughout the cage. Injection of $100 \mu \mathrm{g}$ of lidocaine or procaine produced no significant change in activity.

\section{Ratio of stereotyped behavior}

As noted above, a high degree of stereotypy was observed following injection of $100 \mu \mathrm{g}$ cocaine into the ventrolateral striatum. This behavior appeared qualitatively different from the cocaine-induced activation following administration into the nucleus accumbens or anterior dorsal striatum. Whereas animals in the NAcc and ADS groups tended to distribute their activity throughout the cage, animals in the VLS group remained relatively stationary. A comparison of the stereotypy ratio between the nucleus accumbens, the anterior dorsal striatum, and the ventrolateral striatum revealed a significant difference in the ratio between the groups $(F(2,17)=4.2, p<0.03)$. While the ADS ratio was not significantly different from that of the nucleus

\section{A. Total activity - N.Acc.}

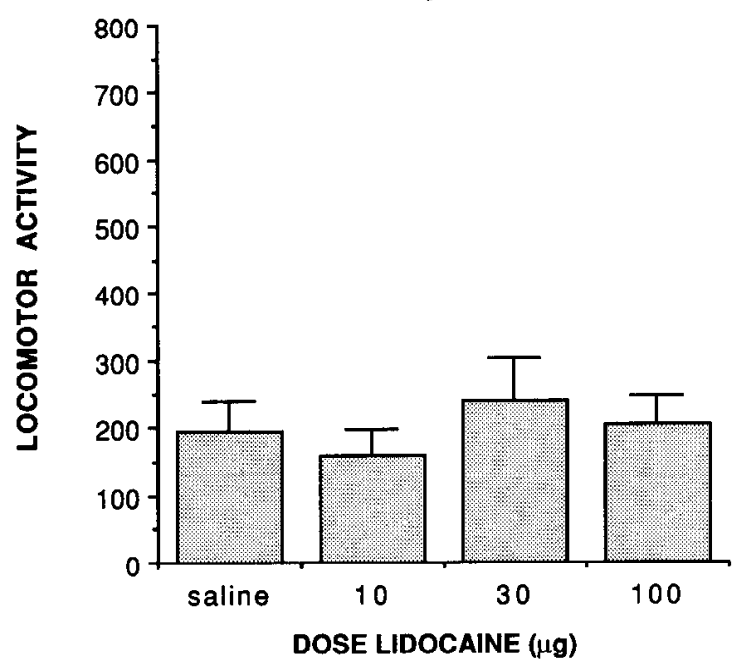

B. Time course - N.Acc.

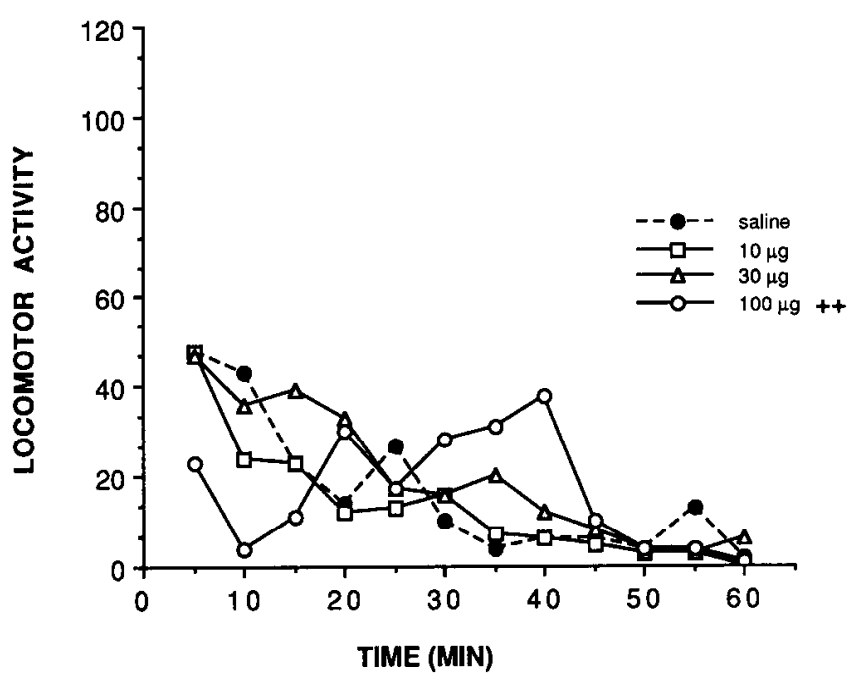

Figure 4. A, Total locomotor activity following microinjection of lidocaine into nucleus accumbens. $B$, Pattern of activity over time, following nucleus accumbens injection of lidocaine.,$++ p<0.01$, dose $x$ time interaction with respect to saline.

accumbens, VLS administration of cocaine produced a significantly higher ratio of stereotypy ( $p<0.05$ ), compared with nucleus accumbens administration. The mean ratios for the sites are shown in Table 1.

\section{Histology}

Examination of cannula placements in brain sections indicated that in all cases the cannula tracks fell in the desired brain regions. Figure 6 shows photomicrographs of representative placements for the nucleus accumbens, anterior dorsal striatum, and ventrolateral striatum.

\section{Discussion}

The present study was designed to compare the behavioral response to cocaine injected directly into the nucleus accumbens with 2 related compounds, procaine and lidocaine. It has been demonstrated, to our knowledge for the first time, that behav- 


\section{A. Total activity - ADS}

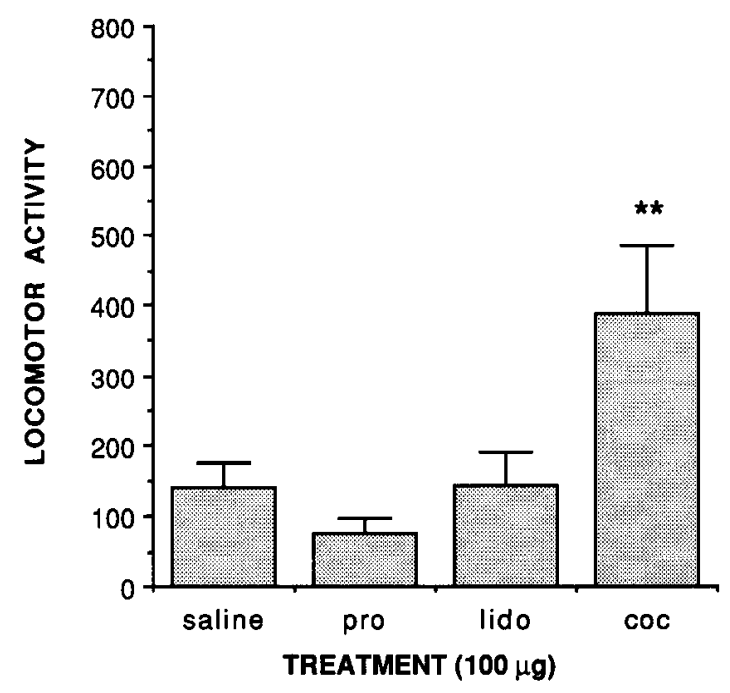

B. TIme course - ADS

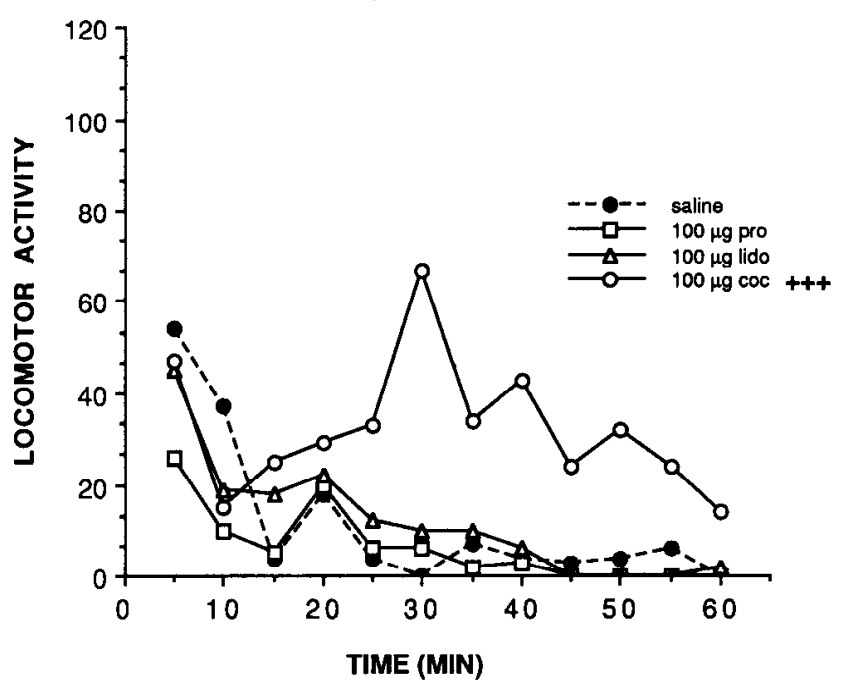

C. Total activity - VLS

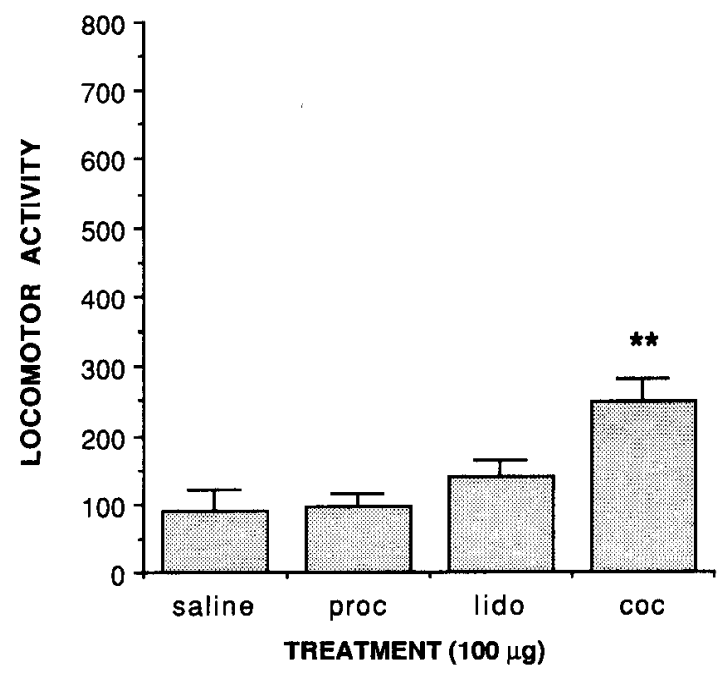

D. TIme course - VLS

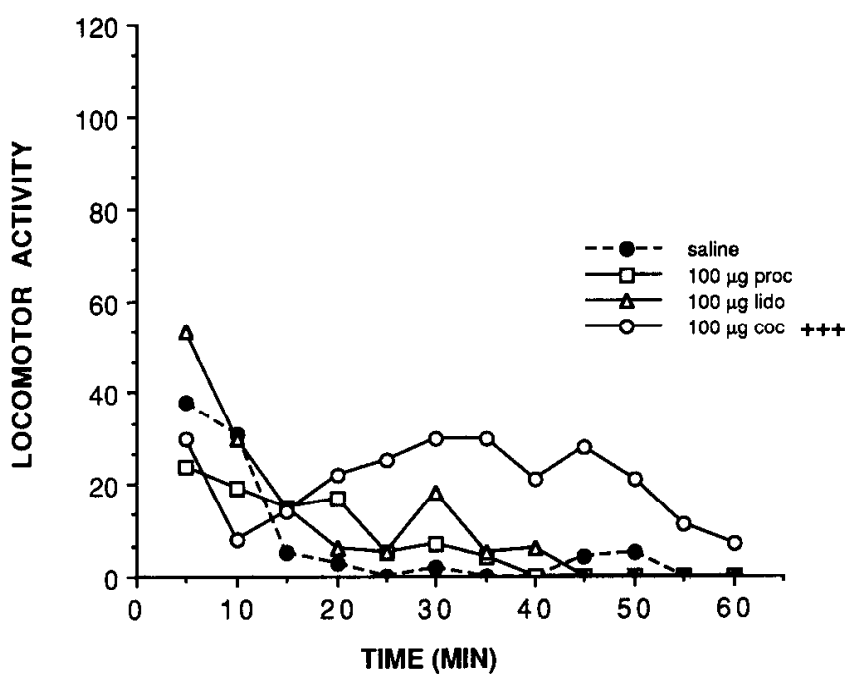

Figure 5. A, Total locomotor activity following injection of procaine, lidocaine, and cocaine (100 $\mu \mathrm{g}$ of each) into the anterior dorsal striatum (ADS). ${ }^{* *} p<0.01$. B. Time course of activity following the 3 ADS treatments.,$+++ p<0.001$, treatment $x$ time interaction with respect to saline. $C$, Total locomotor activity following injection of procaine, lidocaine, or cocaine into ventrolateral striatum (VLS). ${ }^{* *} p<0.01 . D$, Time course of activity following the 3 VLS treatments.,$+++ p<0.001$, treatments $\times$ time interaction with respect to saline.

ioral activation results from direct intracerebral microinjection of cocaine. The results are consistent with the hypothesis that enhancement of DA in the region of the nucleus accumbens mediates the behaviorally activating properties of cocaine. Considering the extensive research relating cocaine to DA function, it is perhaps surprising that investigators have not previously examined the behavioral effects of cocaine microinjection into the nucleus accumbens.

The profiles of locomotor activity following administration of the various compounds into the nucleus accumbens were strongly differentiated. Cocaine elicited a marked, dose-dependent increase in activity which lasted approximately $1 \mathrm{hr}$. It is unlikely that this behavioral effect is due to local anesthetic properties of cocaine, since neither procaine nor lidocaine induced clear increases in activity. Procaine and lidocaine actually decreased activity initially. This decrease was followed by a small, but significant rise in activity later in the session. It may be that the inhibition (particularly apparent with lidocaine) is due to local anesthetic effects, and that the mild activation following the inhibition is due to compensatory alterations in neuronal activity. Evidence that the cocaine-induced activity is mediated by DA is provided by the finding that concurrent administration of a neuroleptic blocked this effect.

The differentiation between cocaine and local anesthetics is supported by many studies. In tests comparing the subjective effects of cocaine and lidocaine, humans discriminated between the 2 and reported lidocaine as similar to saline (Fischman et al., 1983). Procaine was also differentiated from cocaine, although in high doses it was rated similar to cocaine. In animal studies, lidocaine is not self-administered; procaine is self-administered although it is a much weaker reinforcer than cocaine (Ford and Balster, 1977; Woolverton and Balster, 1979; Johan- 


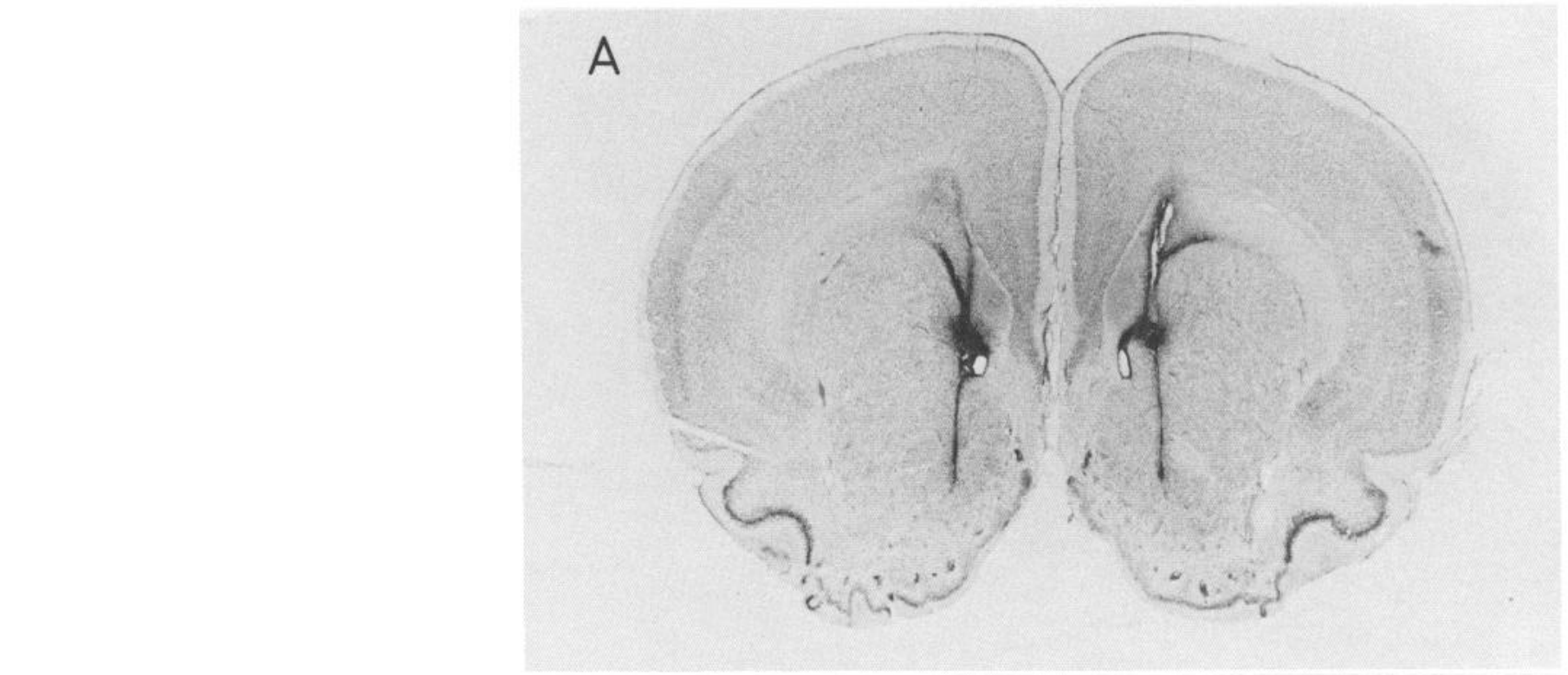

Figure 6. Photomicrographs of selected coronal Nissl-stained sections showing cannulae placements. $A, \mathrm{Nu}$ cleus accumbens. $B$, Anterior dorsal striatum. $C$, Ventrolateral striatum.

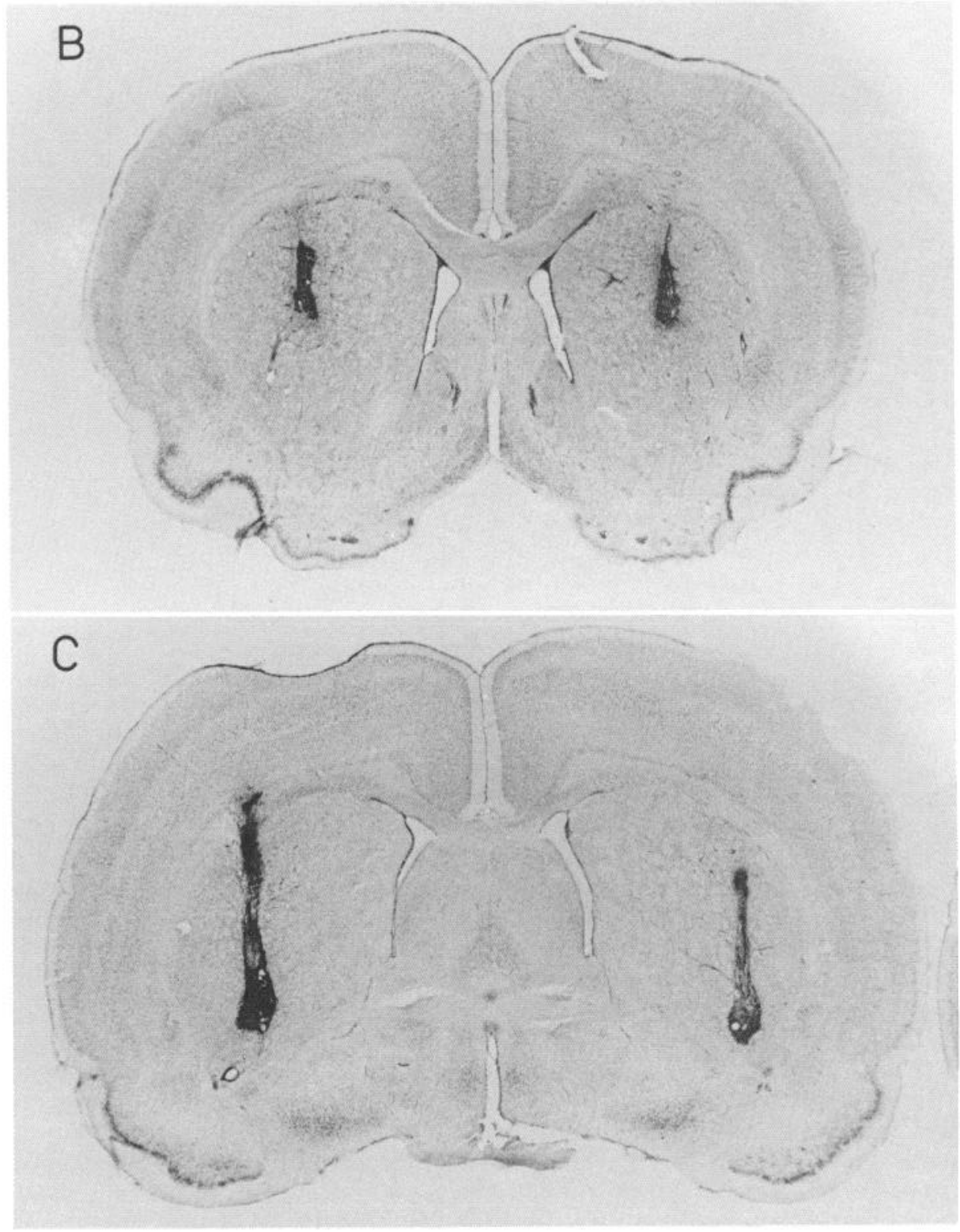


Table 1. Stereotypy ratio following cocaine microinjection $(100 \mu \mathrm{g})$ into dopaminergic sites

\begin{tabular}{ll} 
Striatal site & $\begin{array}{l}\text { Stereotypy ratio } \\
\text { (total activity/ } \\
\text { locomotor counts) }\end{array}$ \\
\hline Nucleus accumbens & $3.7 \pm 0.32$ \\
Anterior dorsal striatum & $4.4 \pm 0.47$ \\
Ventrolateral striatum & $6.0 \pm 0.70^{b}$
\end{tabular}

"See Materials and Methods for definition.

" $p<0.05$, Newman-Keuls test (compared with nucleus accumbens).

son, 1980; Johanson and Aigner, 1981). Although it can act as a reinforcer, evidence suggests that procaine exerts its discriminative and reinforcing effects through mechanisms other than inhibition of the DA uptake site (De La Garza and Johanson, 1982; Morency and Beninger, 1986).

In contrast, there is ample evidence that cocaine exerts its psychostimulant and rewarding effects through stimulation of the DA system. Pharmacological blockade of the dopaminergic systems with DA receptor antagonists during cocaine selfadministration selectively decreases the reinforcement strength of the drug (Wilson and Shuster, 1972; De La Garza and Johanson, 1982; Ettenberg et al., 1982). Although it is generally accepted that DA mediates cocaine self-administration, there is lack of agreement concerning which brain site(s) are involved. Selective lesions of the mesolimbic DA cell bodies disrupt cocaine self-administration (Roberts and Koob, 1982). Lesions of the DA terminals in the nucleus accumbens with 6-hydroxydopamine, as well as lesions of cell bodies in the accumbens, result in attenuation or extinction of intravenous cocaine selfadministration (Roberts et al., 1980; Zito et al., 1985), suggesting that this structure is the site underlying cocaine's reinforcing effects. Microdialysis techniques have shown that both systemic and intracerebral (nucleus accumbens) injections of amphetamine and cocaine significantly increase extracellular DA in the nucleus accumbens (DiChiara and Imperato, 1988; Hernandez and Hoebel, 1988). However, Goeders and Smith (1983) reported that rats would acquire and maintain self-administration for cocaine directly into the medial prefrontal cortex (mPFC), but not into the nucleus accumbens. This is surprising in view of the fact that both amphetamine and DA are self-injected into the nucleus accumbens (Hoebel et al., 1983; Hernandez and Hoebel, 1988). Goeders and Smith also reported that 6-hydroxydopamine lesions of DA terminals at the prefrontal selfadministration site abolished responding (1986). In contrast to this finding, 6-OHDA lesions of the mPFC have no effect on intravenous self-administration, suggesting that intravenous cocaine self-administration is not dependent on innervation of the mPFC (Martin-Iverson et al., 1986). Although the present results have not examined the rewarding effects of cocaine, they support the view that the motor-activating properties of cocaine are mediated by the nucleus accumbens.

Perhaps the strongest evidence linking cocaine reinforcement to DA is provided by the studies of Ritz et al. (1987), who found a strong correlation between the ability of cocaine-like compounds to bind to the DA uptake site and their potency in selfadministration. For example, procaine's relative potency at the DA uptake site is 164 -fold weaker than that of cocaine, while the relative potency of lidocaine is 5,153 time weaker than that of cocaine. Cocaine is readily self-administered, procaine is selfadministered only in high doses, and lidocaine is not self-administered. These findings suggest that the lack of motor activation following procaine and lidocaine infusion into the nucleus accumbens is due to a weak affinity for the DA transporter.

The effects of drug infusion into the 2 neighboring striatal sites require careful consideration with regard to the problem of diffusion. It is clear from the results that the motor stimulation induced by cocaine injection into nucleus accumbens is not entirely site-specific, as significant increases in behavior resulted from anterior dorsal or ventrolateral striatal injections. It is possible, and likely, that diffusion of injected cocaine away from these sites toward the nucleus accumbens region contributed to the activating effect. Although an autoradiographic study would be required to ascertain definitively the amount of spread, cocaine is highly lipophilic and spreads rapidly through tissues. The profile of activity following ADS and VLS cocaine injections, with the greatest effect occurring later in the test session, supports a diffusion hypothesis. Further evidence for diffusion is provided by the fact that in the nucleus accumbens, the high dose of cocaine produced a 5-fold increase in activity, whereas it produced a 3-fold and 2-fold increase in the ADS and VLS, respectively. In contrast to the results with cocaine, this laboratory has found a high degree of anatomical specificity with regard to the behavioral effects of amphetamine microinjections into different regions of striatum (Kelley et al., 1988, 1989). For example, amphetamine microinjected in high doses into the VLS produces intense oral stereotypy accompanied by a decrease in locomotion. In contrast, cocaine injected into this site produced no biting or licking and increased locomotion. However, the fact that more stationary, perseverative movement was elicited by cocaine at this site nevertheless supports the idea that this site mediates the stereotypic effects of stimulants, and not their locomotor activating properties.

As mentioned previously, there is little information on effects of cocaine directly microinjected into the brain. There is one report of cocaine microinjected into the cerebral ventricles (Elliott et al., 1987). Surprisingly, these investigators reported no motor activation following ventricular injection of $100 \mu \mathrm{g}$ cocaine, although they did report the well-known hyperactivity following systemic administration. It is possible that cocaine diffuses so quickly that concentrations that eventually reach the nucleus accumbens are not high enough to induce behavioral activation. Morency and co-workers found that unilateral injection of cocaine into the medial prefrontal cortex induced contraversive circling behavior (Morency et al., 1987). Procaine, even in doses as high as $1000 \mu \mathrm{g}$, did not induce circling. These authors suggest that the mPFC is involved in the control of locomotion, although other sites were not studied. They believe that diffusion from the frontal cortex to the nucleus accumbens is unlikely; however, in view of our findings, it is quite possible that diffusion could occur.

It is concluded that cocaine infusion into the nucleus accumbens clicits a pronounced motor activation, which is not duc to the local anesthetic properties of the drug, and which is mediated by increased synaptic DA. Behavior elicited by direct microinjections into the forebrain DA system can now be further explored in paradigms that test for effects on reinforcement. Such studies may help to elucidate the relative contributions of different dopaminergic regions to the rewarding and addictive properties of cocaine. 


\section{References}

Bruning, J. L., and B. L. Kintz (1977) Computational Handbook of Statistics, Scott, Foresman, Glenview, IL.

Carr, G. D., and N. White (1987) Effects of systemic and intracranial amphetamine injections on behavior in the open field: A detailed analysis. Pharmacol. Biochem. Behav. 27: 113-122.

Collins, R. J., J. R. Weeks, M. M. Cooper, P. I. Good, and R. R. Russel (1984) Prediction of abuse liability of drugs using IV self-administration by rats. Psychopharmacology 82: 6-13.

De La Garza, R., and C. E. Johanson (1982) Effects of haloperidol and physostigmine on self-administration of local anesthetics. Pharmacol. Biochem. Behav. 17: 1295-1299.

Di Chiara, G., and A. Imperato (1988) Drugs abused by humans preferentially increase synaptic dopamine concentrations in the mesolimbic system of freely moving rats. Proc. Natl. Acad. Sci. USA 85: 5274-5278.

Elliott, P. J., G. M. Rosen, and C. B. Nemeroff (1987) A comparison of cocaine and its metabolite norcocaine: Effects on locomotor activity. Pharmacol. Biochem. Behav. 26: $573-575$.

Ettenberg, A., H. O. Pettit, F. E. Bloom, and G. F. Koob (1982) Heroin and cocaine intravenous self-administration in rats: Mediation by separate neural systems. Psychopharmacology 78: 204-209.

Fischman, M. W., C. R. Schuster, and S. Rajfer (1983) A comparison of the subjective and cardiovascular effects of cocaine and procaine in humans. Pharmacol. Biochem. Behav. 18: 711-716.

Ford, R. D., and R. L. Balster (1977) Reinforcing properties of intravenous procaine in rhesus monkeys. Pharmacol. Biochem. Behav. 6 : 289-296.

Goeders, N. E., and J. E. Smith (1983) Cortical dopaminergic involvement in cocaine reinforcement. Science 221: 773-775.

Goeders, N. E., and J. E. Smith (1986) Reinforcing properties of cocaine in the medial prefrontal cortex: Primary action on presynaptic terminals. Pharmacol. Biochem. Behav. 25: 191-199.

Hernandez, L., and B. G. Hoebel (1988) Food reward and cocaine increase extracellular dopamine in the nucleus accumbens as measured by microdialysis. Life Sci. 42: 1705-1712.

Hoebel, B. G., A. P. Monaco, L. Hernandez, E. Aulissi, B. G. Stanley, and L. Lenard (1983) Self-injection of amphetamine directly into the brain. Psychopharmacology 81: 158-163.

Johanson, C. E. (1980) The reinforcing properties of procaine, chlorprocaine, and proparacaine in rhesus monkeys. Psychopharmacology 67: 189-194.

Johanson, C. E., and T. Aigner (1981) Comparison of the reinforcing propertics of cocaine and procainc in rhesus monkeys. Pharmacol. Biochem. Behav. 15: 49-53.

Kelley, A. E., and A. M. Gauthier (1987) Differential effects on spontaneous motor behavior, feeding, and oral stereotypy following amphetamine microinjections into anatomically defined subregions of rat striatum. Soc. Neurosci. Abstr. 13: 31.

Kelley, A. E., C. G. Lang, and A. M. Gauthier (1988) Induction of oral stereotypy following amphetamine microinjection into a discrete subregion of the striatum. Psychopharmacology 95: 556-559.

Kelley, A. E., A. M. Gauthier, and C. G. Lang (1989) Amphetamine microinjections into distinct striatal subregions cause dissociable effects on motor and ingestive behavior. Behav. Brain Res. 35: 27-39.

Kelly, P. H., and S. D. Iversen (1976) Selective 6-OHDA induced destruction of mesolimbic dopamine neurons: Abolition of psychostimulant-induced locomotor activity in rats. Eur. J. Pharmacol. 40: $45-56$.
Kelly, P. H., P. W. Seviour, and S. D. Iversen (1975) Amphetamine and apomorphine responses in the rat following 6-OHDA lesions of the nucleus accumbens septi and corpus striatum. Brain Res. 94:507522 .

Martin-Iverson, M. T., C. Szostak, and H. C. Fibiger (1986) 6-Hydroxydopamine lesions of the medial prefrontal cortex fail to influence intravenous self-administration of cocaine. Psychopharmacology 88 . 310-314.

Morency, M. A., and R. J. Beninger (1986) Dopaminergic substrates of cocaine-induced place conditioning. Brain Res. 399: 33-41.

Morency, M. A., R. J. Stewart, and R. J. Beninger (1987) Circling behavior following unilateral microinjections of cocaine into the medial prefrontal cortex: dopaminergic or local anesthetic effect? J. Neurosci. 7: 812-818.

Pettit, H. O., A. Ettenberg, F. E. Bloom, and G. F. Koob (1984) Destruction of dopamine in the nucleus accumbens selectively attenuates cocaine but not heroin self-administration in rats. Psychopharmacology 84: 167-173.

Pickens, R., and T. Thompson (1968) Cocaine-reinforced behavior in rats: Effects of reinforcement magnitude and fixed ratio size. J. Pharmacol. Exp. Ther. 161: 122-129.

Pijnenburg, A. J. J., W. M. M. Honig, J. A. M. Van der Heyden, and J. M. Van Rossum (1976) Effects of chemical stimulation of the mesolimbic dopamine system upon locomotor activity. Eur. J. Pharmacol. 35: 45-58.

Post, R. M., S. R. B. Weiss, A. Pert, and T. W. Uhde (1987) Chronic cocaine administration: sensitization and kindling effects. In Cocaine: Clinical and Biobehavioral Aspects, S. Fishcr, A. Raskin, and E. H. Uhlenhuth, eds., pp. 109-173, Oxford U. P., New York.

Reith, M. E. A., H. Sershen, and A. Lajtha (1980) Saturable [3H]cocaine binding in central nervous system of mouse. Life Sci. 27 . 1055-1062.

Ritz, M. C., R. J. Lamb, S. R. Goldberg, and M. J. Kuhar (1987) Cocaine receptors on dopamine transporters are related to self-administration of cocaine. Science 237: 1219-1223.

Roberts, D. C. S., and G. F. Koob (1982) Disruption of cocaine selfadministration following 6-hydroxydopamine lesions of the ventral tegmental area in rats. Pharmacol. Biochem. Behav. 17: 901-904.

Roberts, D. C. S., M. E. Corcoran, and H. C. Fibiger (1977) On the role of ascending catecholaminergic systems in intravenous selfadministration of cocaine. Pharmacol. Biochem. Behav. 6: 615-620.

Roberts, D. C. S., G. F. Koob, P. Klonoff, and H. C. Fibiger (1980) Extinction and recovery of cocaine self-administration following 6 hydroxydopamine lesions of the nucleus accumbens. Pharmacol. Biochem. Behav. 12: 781-787.

Schuster, C. R., and T. Thompson (1969) Self-administration of and behavioral dependence on drugs. Annu. Rev. Pharmacol. 9: 483-502.

Wilson, M. C., and C. R. Schuster (1972) The effects of chlorpromazine on psychomotor stimulant self-administration in the rhesus monkey. Psychopharmacologia 26: 115-126.

Woolverton, W. L., and R. L. Balster (1979) Reinforcing properties of some local anesthetics in rhesus monkeys. Pharmacol. Biochem. Behav. 11: 669-672.

Zito, K. A., G. Vickers, and D. C. S. Roberts (1985) Disruption of cocaine and heroin self-administration following kainic acid lesions of the nucleus accumbens. Pharmacol. Biochem. Bchav. 23: 10291036. 